\title{
RAMAN SPECTROSCOPY OF AMORPHOUS CARBON
}

$$
\text { SAND- }-98-01280
$$

\author{
D. R. TALLANT, T. A. FRIEDMANN, N. A. MISSERT, M. P. SIEGAL AND J. P. \\ SULLIVAN
}

Sandia National Laboratories, P.O. Box 5800, Albuquerque, NM 87185-1411, drtalla@sandia.gov

$$
\text { CONF- } 971201-
$$

RECEIVED

JAN 291998

ABSTRACT

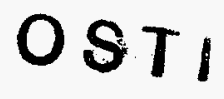

Amorphous carbon is an elemental form of carbon with low hydrogen content, which may be deposited in thin films by the impact of high energy carbon atoms or ions. It is structurally distinct from the more well-known elemental forms of carbon, diamond and graphite. It is distinct in physical and chemical properties from the material known as diamond-like carbon, a form which is also amorphous but which has a higher hydrogen content, typically near 40 atomic percent. Amorphous carbon also has distinctive Raman spectra, whose patterns depend, through resonance enhancement effects, not only on deposition conditions but also on the wavelength selected for Raman excitation. This paper provides an overview of the Raman spectroscopy of amorphous carbon and describes how Raman spectral patterns correlate to film deposition conditions, physical properties and molecular level structure.

\section{INTRODUCTION}

The elemental forms of carbon include a range of structures whose end members are diamond and graphite. In diamond the carbon atoms are four-coordinated and connected by bonds with $\sigma$-character (single bond order) in an $\mathrm{sp}^{3}$ (tetrahedral) configuration. Graphite is composed of stacked, planar sheets of six-membered rings of three-coordinated carbon. Graphitic carbon has conjugated single and double bonds in a ( $\pi$-bonded) $\mathrm{sp}^{2}$ configuration. The stacking of planar sheets of six-membered rings is integral to the structure of graphite and affects both its properties and its spectroscopic signature.

Carbon films deposited by the impact of high energy carbon atoms or ions, which are accelerated from a graphite target by pulsed laser ablation [1, 2] or a filtered arc [3], can form an amorphous matrix with a complex molecular structure. First principles theoretical simulations of this amorphous carbon film (also known as amorphous tetrahedral carbon [4-6]) predict a rich variety of hybridized bonding which is not like that of diamond $\left(\mathrm{sp}^{3}\right)$ or graphite $\left(\mathrm{sp}^{2}\right)$ but includes three-fold coordinated and four-fold coordinated carbon atoms. For depositions utilizing higher energy excitation conditions, the four-coordinated carbon atoms are in the majority. Estimates of four-coordinated carbon comprising up to $80 \%$ of the total species have been made (see, e.g., [7]).

Raman spectra of elemental carbon reflect the differences in bonding of the different forms. Figure 1 shows the Raman spectra of crystalline diamond, an amorphous carbon film, nanocrystalline graphite and graphite with microscale to macroscale crystalline domains. These spectra were obtained using excitation at visible wavelengths. Diamond has a single Raman band in this frequency region, peaking at
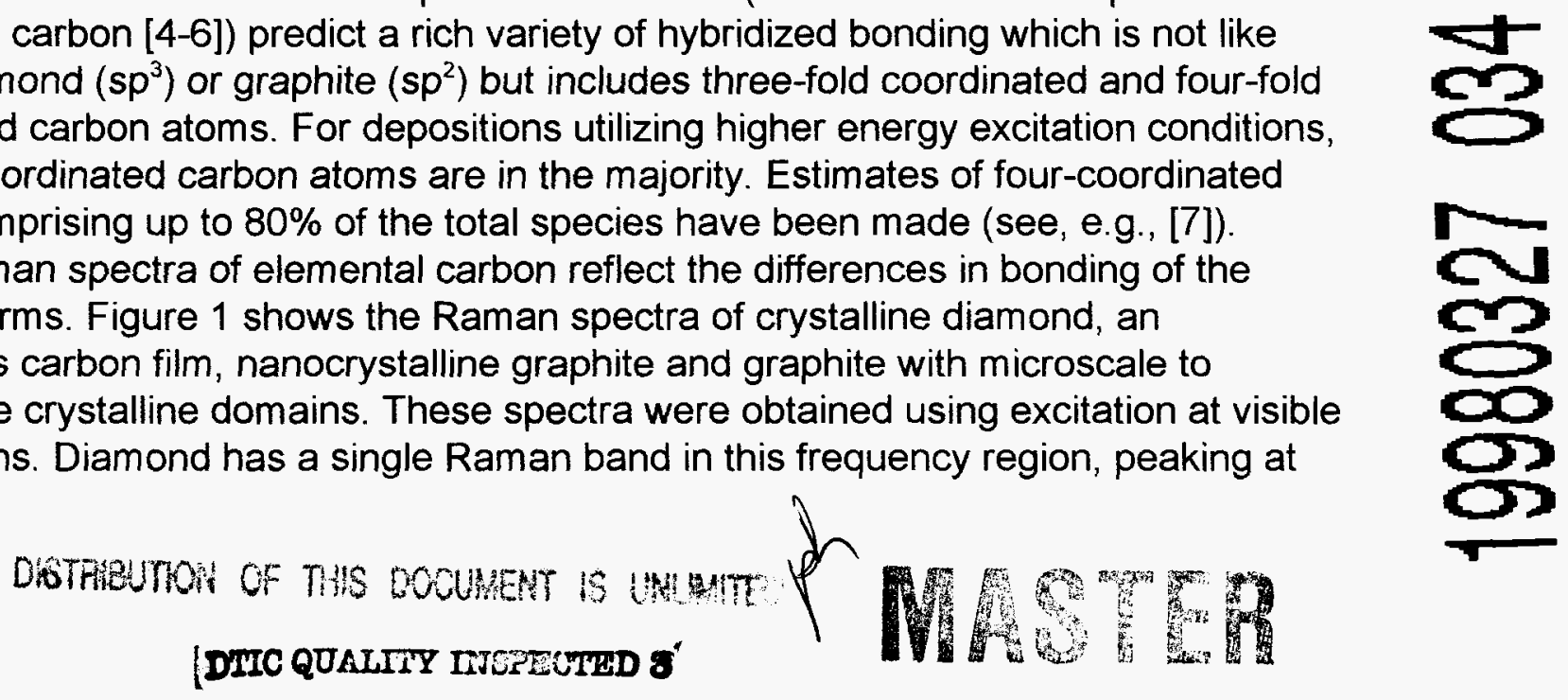


\section{DISCLAIMER}

This report was prepared as an account of work sponsored by an agency of the United States Government. Neither the United States Government nor any agency thereof, nor any of their employees, makes any wartanty, express or implied, or assumes any legal liability or responsibility for the accuracy, completeness, or usefulness of any information, apparatus, product, or process disclosed, or represents that its use would not infringe privately owned rights. Reference herein to any specific commercial product, process, or service by trade name, trademark, manufacturer, or otherwise does not necessarily constitute or imply its endorsement, recommendation, or favoring by the United States Government or any agency thereof. The views and opinions of authors expressed herein do not necessarily state or reflect those of the United States Government or any agency thereof. 
$1332 \mathrm{~cm}^{-1}$. Graphite has a Raman band due to a Brillouin-zone-center phonon at $1578 \mathrm{~cm}^{-1}$ (the "G" band) and a Raman band peaking near $1350 \mathrm{~cm}^{-1}$ (the "D" band), which is due to a zone-edge phonon that becomes symmetry-allowed in the reducedsymmetry environment at the edges of graphite crystals [8]. The Raman bands of graphite are enhanced in intensity with visible excitation by the interaction of electronic $\left(\pi \rightarrow \pi^{*}\right)$ transitions with vibrational transitions, such that Raman spectra of submicrometer thicknesses of graphite are easily obtained. Diamond Raman bands do not experience resonance enhancement with visible excitation, since vacuum ultraviolet wavelengths are required to access the $\left(\sigma \rightarrow \sigma^{*}\right)$ electronic transitions of diamond.

Graphite retains its structure of stacked planes of six-membered carbon rings even with domain sizes down to nanometer-scale dimensions, and the Raman spectra of "nanocrystalline" graphite show broadened bands and enhanced intensity in the " $D$ " band [9]. The double-banded structure shown in the Raman spectrum of Figure 1 is characteristic of graphite with nanoscale domains.

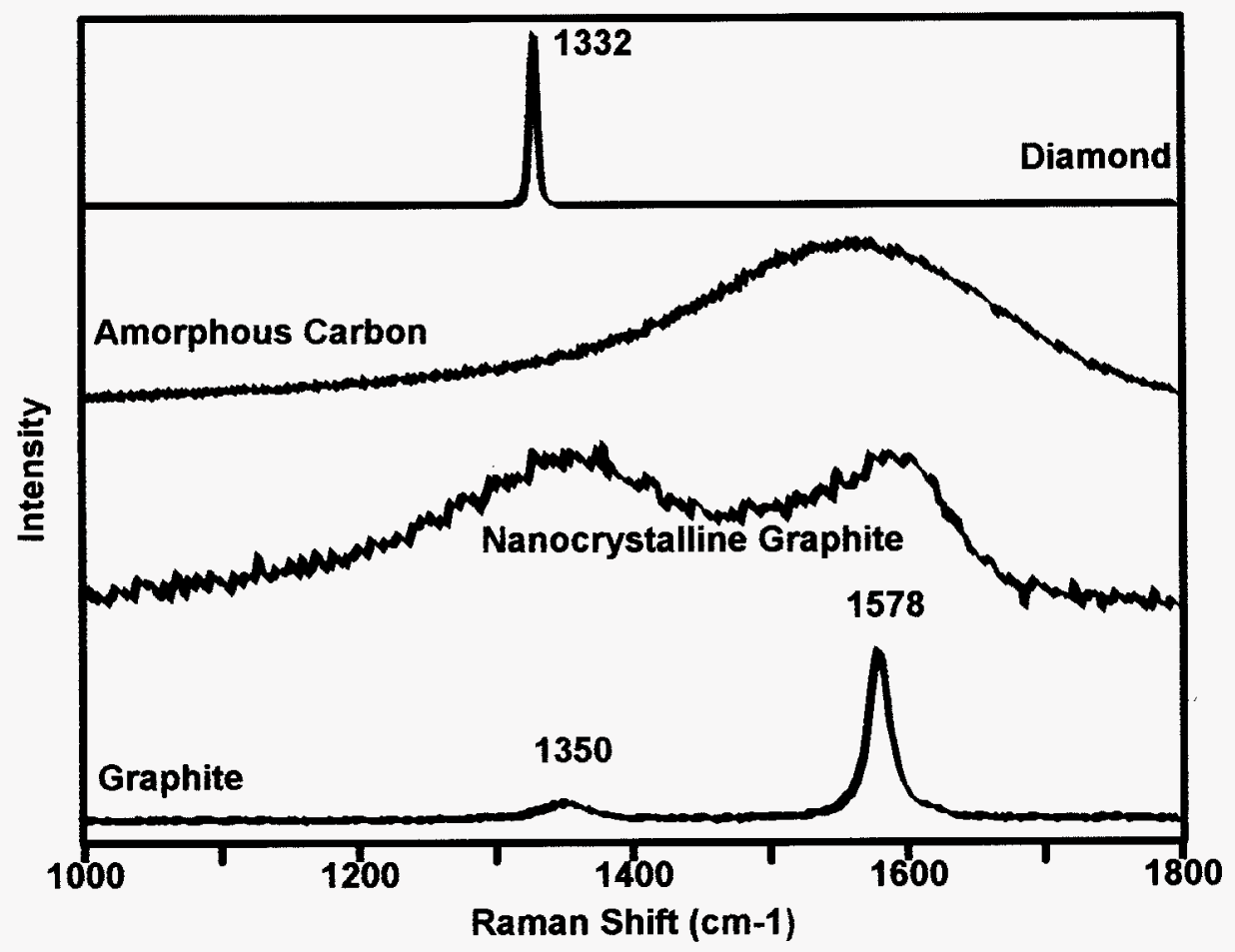

Figure 1. Raman spectra of forms of elemental carbon

Vibrational bands from three-coordinated ( $\pi$-bonded) carbon structures dominate the Raman spectra of amorphous carbon when visible wavelengths are used for excitation. As with graphite, these three-coordinated carbon structures experience resonance enhancement of their Raman band intensities. However, the characteristic, two-band Raman pattern of graphite is generally not present in the Raman spectra of amorphous carbon (see Figure 1). Rather there is a broad band of complex shape, peaking from 1500 to $1570 \mathrm{~cm}^{-1}$, whose peak position and shape vary with deposition conditions. There appear to be a multiplicity of three-coordinated carbon structures 
contributing to the Raman spectra, whose distribution varies in relation to the properties of the amorphous carbon films.

There is less resonance enhancement of the three-coordinated carbon structures with ultraviolet excitation. Raman spectra of amorphous carbon films show enhanced intensity in a broad band peaking at 1100 to $1200 \mathrm{~cm}^{-1}$, which has been interpreted as being due to four-coordinated carbon structures [7, 10]. Apparently, the reduction in intensity of the Raman bands due to three-coordinated carbon structures permits the observation of Raman bands due to four-coordinated carbon structures. Thus, Raman with ultraviolet excitation is useful for detecting differences in the ratio of four-coordinated to three-coordinated carbon structures in amorphous carbon.

\section{EXPERIMENT}

The carbon films whose spectra are displayed in this paper were deposited on silicon wafer substrates by pulsed $(248 \mathrm{~nm})$ laser ablation off a graphite target in vacuum [2]. Raman spectra were obtained using an argon ion laser for excitation (visible and near ultraviolet wavelengths). A triple spectrograph and charge-coupleddevice detector were used to record the Raman spectra.

\section{RAMAN WITH VISIBLE EXCITATION}

Figure 2 shows Raman spectra, excited with 514-nm laser light, of a series of amorphous carbon films, all approximately $100 \mathrm{~nm}$ thick, which were deposited with laser ablation energies from 5 to $125 \mathrm{~J} / \mathrm{cm}^{2}$. Higher deposition energies are believed to favor the formation of four-coordinated carbon [11]. In addition to a broad carbon Raman band peaking between 1500 and $1600 \mathrm{~cm}^{-1}$, there is a flat-topped Raman feature between 900 and $1000 \mathrm{~cm}^{-1}$, which is a second-order phonon band from the silicon substrate. The silicon Raman band increases in intensity with increasing deposition energy. The increase in silicon Raman band intensity implies that the films become more transparent as the deposition energy increases. The reason for this increase in transparency is believed to be a decrease in three-coordinated carbon structures with increasing deposition energy, resulting in less absorption of visible light by $\pi \rightarrow \pi^{\star}$ electronic transitions. The carbon Raman band, which is due to structures incorporating three-coordinated carbon atoms, shifts to higher frequency and becomes narrower and more symmetric with increasing deposition energy. Its integrated intensity decreases as the proportion of three-coordinated carbon atoms decreases. Increasing deposition energy appears not only to reduce the overall proportion of threecoordinated carbon atoms but also to narrow the distribution of structures in which they reside, resulting in a smaller spread of vibrational energies.

Film thickness also affects the appearance of Raman spectra of amorphous carbon. In Figure 3 we show spectra of two films, both deposited at $44 \mathrm{~J} / \mathrm{cm}^{2}$, one of which was grown to a thickness of $7 \mathrm{~nm}$ and the other to a thickness of $200 \mathrm{~nm}$. The spectrum of the thicker film has the appearance of the films in Figure 2 grown at moderate-to-high deposition energies. The carbon Raman band is relatively narrow and symmetric and peaks at a relatively high frequency (about $1545 \mathrm{~cm}^{-1}$ ). Films deposited at $44 \mathrm{~J} / \mathrm{cm}^{2}$ and thicker than $50 \mathrm{~nm}$ give spectra like that of the $200-\mathrm{nm}$ thick film. But 


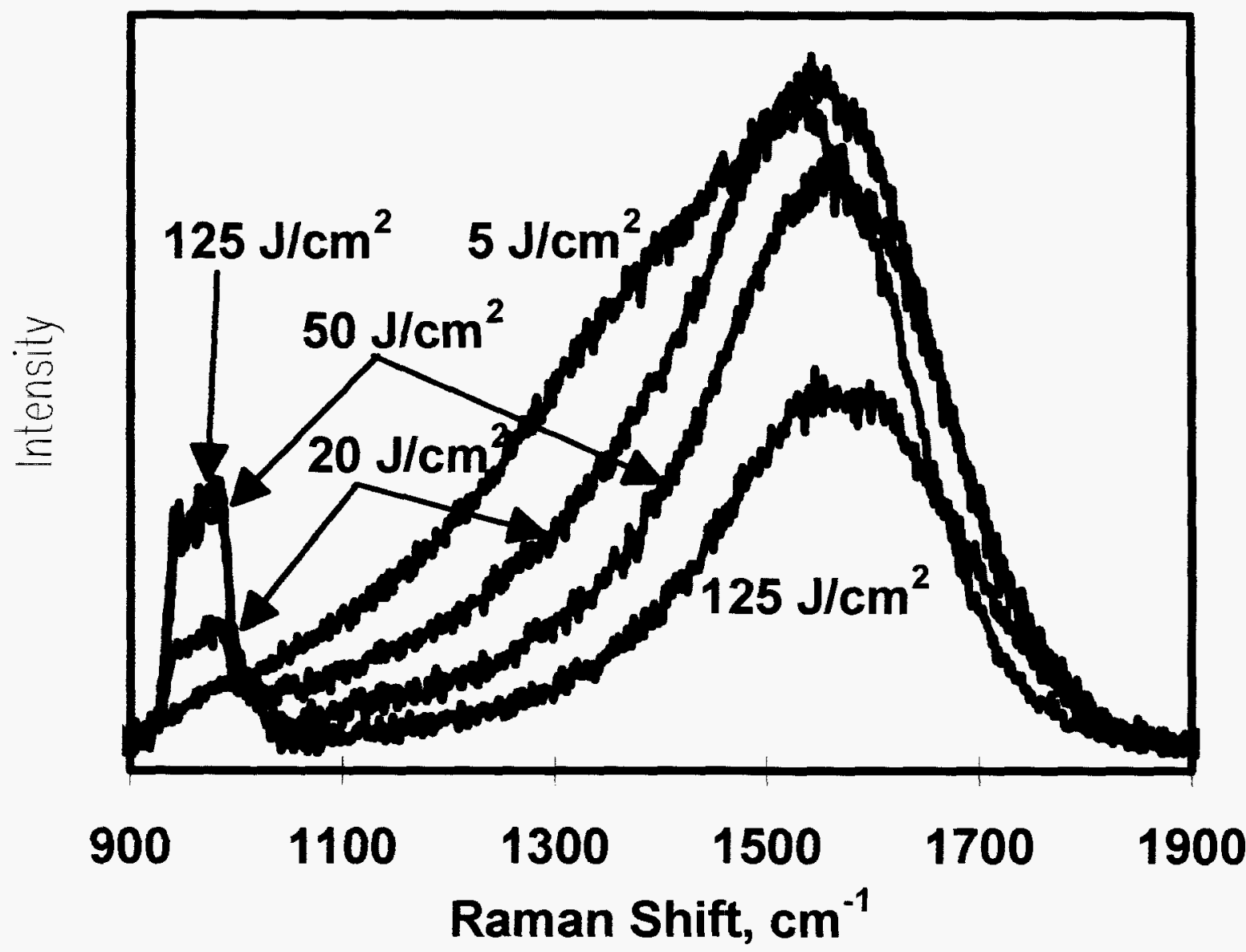

Figure 2. Raman spectra of amorphous carbon films deposited with laser energies as snown on the figure. The Raman excitation wavelength was $514 \mathrm{~nm}$.

the carbon Raman band of the thin $(7 \mathrm{~nm})$ film peaks at a significantly lower frequency $\left(1405 \mathrm{~cm}^{-1}\right)$ and is broadened asymmetrically to lower frequency, much like those carbon Raman bands deposited at lower energies (Figure 2). Clearly the 7-nm film is dominated by an interfacial layer which is different from the bulk of thicker films. The composition of the layer is not known for sure, but it may have a relatively high proportion of three-coordinated carbon atoms, as if it were deposited at a lower deposition energy. Alternately, the incorporation of silicon atoms could explain the downward shift in frequency of the carbon Raman band. Silicon atoms are more massive than carbon, and $\mathrm{Si}-\mathrm{C}$ bonds are less strong than $\mathrm{C}-\mathrm{C}$ bonds. Both higher mass and lower bond strength result in a lower vibrational frequency.

Although three-coordinated structures tend to be in the minority in carbon films, their Raman spectra reflect changes not only in their structures (Figure 2) but also in 


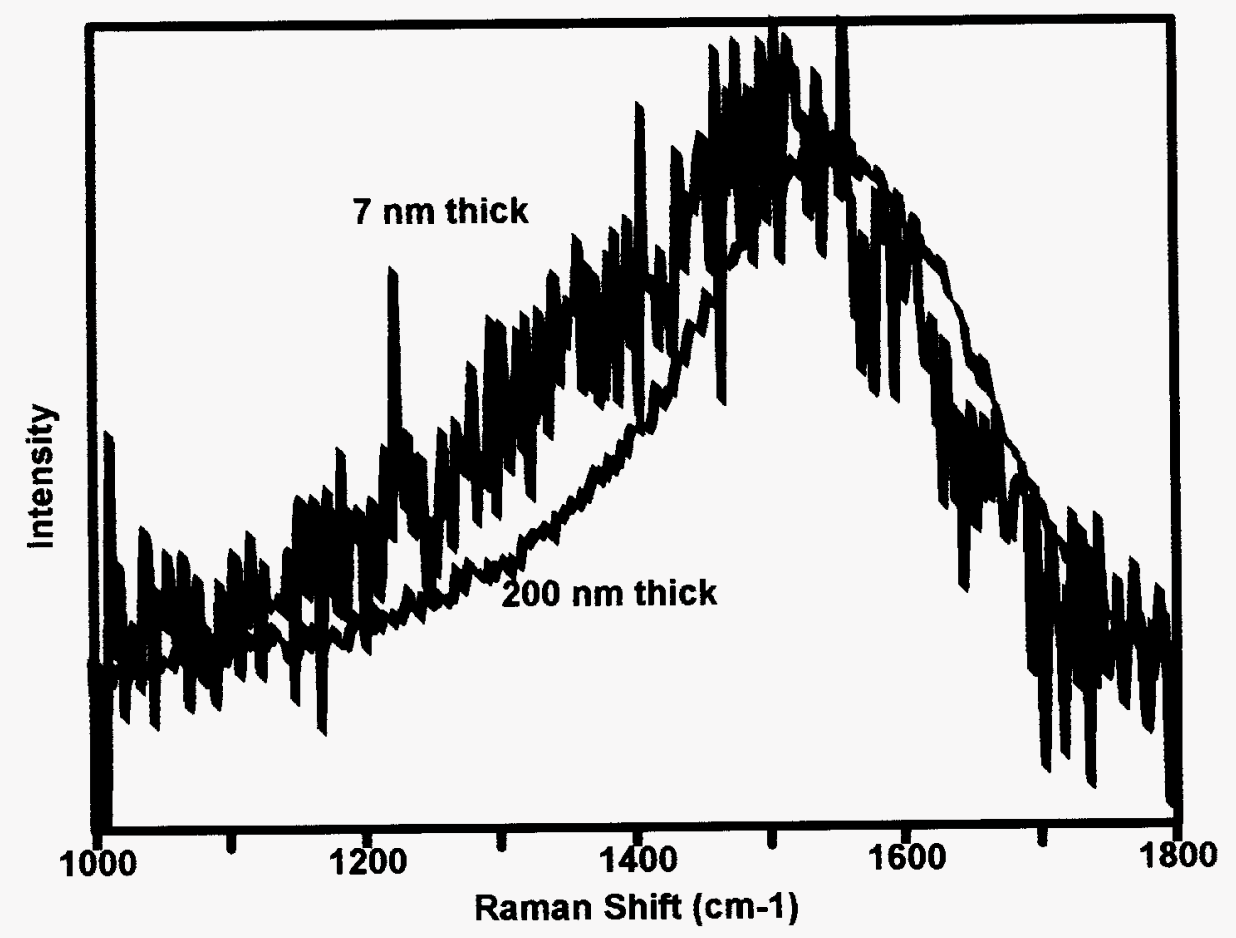

Figure 3. Raman spectra of amorphous carbon films deposited, at $44 \mathrm{~J} / \mathrm{cm}^{2}$, to thicknesses of $7 \mathrm{~nm}$ and $200 \mathrm{~nm}$. The Raman excitation wavelength was $514 \mathrm{~nm}$.

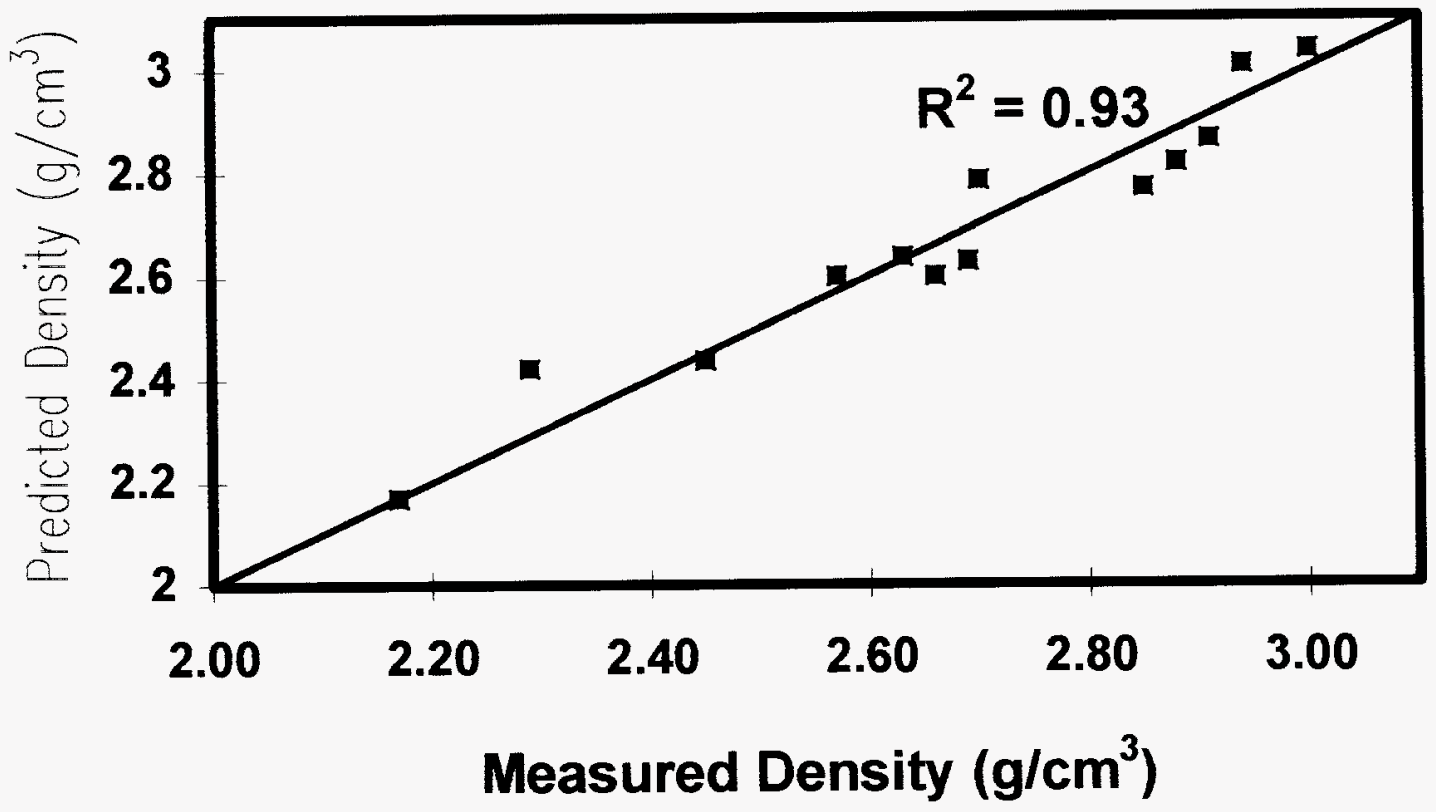

Figure 4. Density of carbon films predicted by Raman spectra versus measured by $x-$ ray diffraction. The prediction was done by multivariate partial least squares analysis. 
the physical properties of the film. In Figure 4 we plot densities of carbon films, as predicted by their Raman spectra, versus densities obtained by fitting glancing angle $x-$ ray reflectivity curves. The carbon films were all about $100 \mathrm{~nm}$ thick. The densities of the films range from 2.2 to $3.0 \mathrm{~g} / \mathrm{cm}^{3}$, generally increasing with deposition energy. The density is believed to increase as the proportion of four-coordinated carbon in the films increases.

The prediction of the densities was performed by multivariate partial least squares (PLS) analysis, which decomposes the Raman spectra into a set of components (like partial spectra) that are common to all the spectra and that correlate to the density. These components are used to reconstruct each Raman spectrum by summing the components with different scaling factors. The combination of spectral components and scaling factors forms a calibration file. The prediction also used cross validation. In cross validation one spectrum is excluded from the set of samples, and a calibration is constructed using the remaining spectra in the set and the measured densities. This "partial" calibration is then used to predict the density of the excluded sample from its Raman spectrum. The process is repeated for all the samples, resulting in a predicted density for each sample. If we plot predicted versus measured densities for each sample in the set (Figure 4), the result is ideally a line with a slope of 1 , passing through the zero intercept, and with all the data points on the line. The correlation coefficient $\left(R^{2}\right.$, which should be close to 1.00$)$ measures how much the points deviate from the ideal line. The $R^{2}=0.93$ for the data in Figure 4 indicates a high correlation between density and the Raman spectra of three-coordinated carbons. A higher correlation $\left(R^{2}=0.98\right)$ was obtained between Raman spectra of the threecoordinated carbon and the as-deposited stress in the film. Stress increases with deposition energy and is believed to also be related to proportion of four-coordinated carbon [11]. Less error is expected in the measurement of the stress than in the density measurement.

Raman spectra obtained with visible excitation wavelengths show changes in the transparency of the film and the distribution of structures incorporating threecoordinated carbon atoms as function of deposition energy. The carbon Raman band patterns from structures incorporating three-coordinated carbon atoms correlate to the density and the stress in the films. Thus, the distribution of structures incorporating three-coordinated carbon atoms reflects, at least to some extent, the overall structure and properties of the carbon film, and their Raman spectra is a useful characterization tool.

\section{RAMAN WITH ULTRAVIOLET EXCITATION}

Shifting the Raman excitation wavelength to ultraviolet wavelengths reduces the resonant enhancement associated with $\pi \rightarrow \pi^{*}$ electronic transitions and decreases the extent to which structures incorporating three-coordinated carbon atoms dominate the Raman spectra. Raman experiments utilizing deep ultraviolet $(244 \mathrm{~nm})$ excitation have shown the appearance, in the Raman spectra, of a broad band, peaking at 1100 to $1200 \mathrm{~cm}^{-1}$, whose intensity correlates with the proportion of four-coordinated carbon atoms in the films $[7,10]$. It is believed that the reduction in the intensity of the resonantly enhanced Raman bands from structures incorporating three-coordinated carbon atoms permits the observation of Raman bands due to four-coordinated carbon 
atoms. It is not yet clear whether the $\sigma \rightarrow \sigma^{*}$ electronic transitions of the four-coordinated carbon atoms extend far enough out of the vacuum ultraviolet to result in significant resonance enhancement with wavelengths above $200 \mathrm{~nm}$.

We have recently developed a capability for ultraviolet Raman and have obtained a Raman spectrum of a carbon film using near ultraviolet ( $364 \mathrm{~nm}$ ) excitation (see Figure 5). Compared to a Raman spectrum of the same film obtained with visible $(514 \mathrm{~nm}$ ) excitation, the near-ultraviolet-excited spectrum shows an additional, broad Raman band extending from $900-1400 \mathrm{~cm}^{-1}$. Also, there is a shift, with ultraviolet excitation, of the three-coordinated carbon band peaking near $1600 \mathrm{~cm}^{-1}$ to higher frequency. Such shifts have been previously reported [7]. These preliminary results suggest that even at this (near) ultraviolet wavelength, suppression of resonance enhancement of the Raman bands from structures incorporating three-coordinated carbon atoms is sufficient to permit observation of a Raman band from four-coordinated carbon atoms.

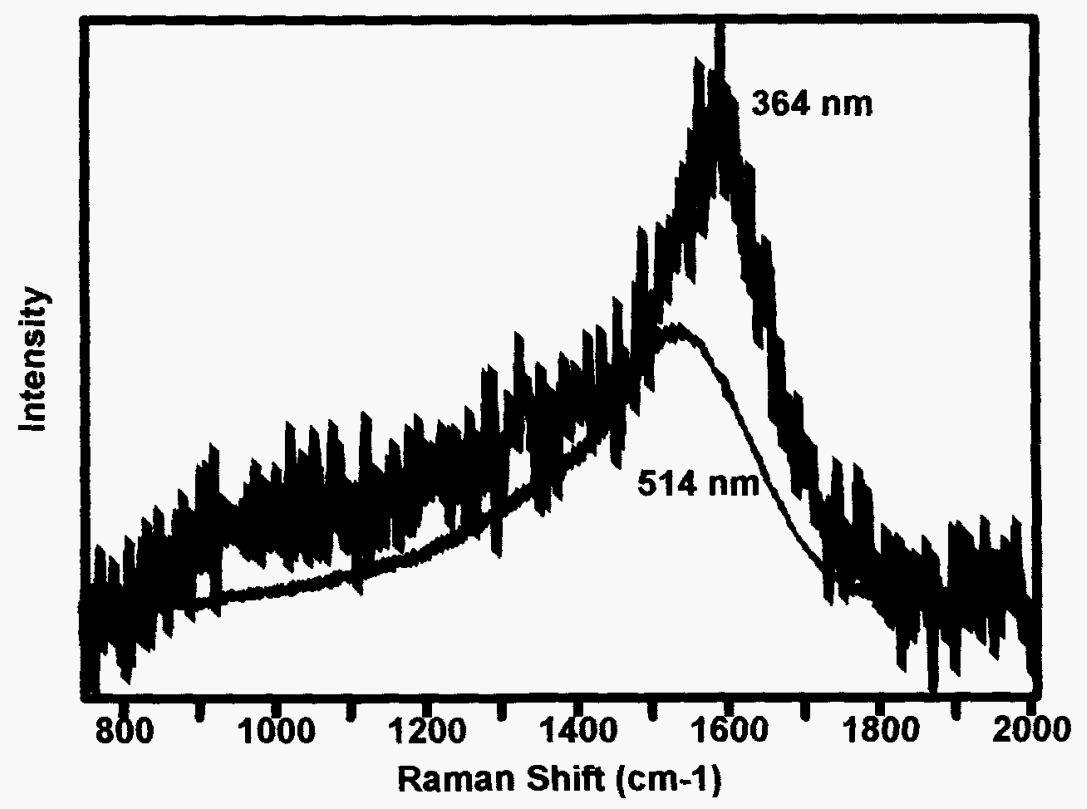

Figure 5. Raman spectra of a carbon film deposited at $5 \mathrm{~J} / \mathrm{cm}^{2}$, with Raman excitation at $364 \mathrm{~nm}$ and $514 \mathrm{~nm}$ as indicated in the figure. Intensities have been scaled.

Using ultraviolet excitation it is possible to record a Raman feature (the band peaking at 1100 to $1200 \mathrm{~cm}^{-1}$ ) which is associated with four coordinated atoms in amorphous carbon films. In the spectra reported thus far, this feature does not appear to shift in peak frequency or change in shape to the same extent as the Raman bands from to three-coordinated carbon structures. However, this Raman band provides a means, through its intensity changes, of directly monitoring changes in the proportion of four-coordinated carbon atoms. 


\section{Four-Coordinated Carbon}

Ultraviolet-excited Raman spectra include a broad band peaking at 1100 to 1200 $\mathrm{cm}^{-1}$, which has been associated with four-coordinated carbon. No definitive experiment has been performed to date to define the structure of the matrix component composed of four-coordinated carbon, but enough is known to suggest its molecular structure.

First of all, it is not a crystalline (or nanocrystalline) form of diamond. Raman spectra of "cluster diamond", a form of crystalline diamond produced by explosive detonation and with a crystal size of about five nanometers, have been reported [12]. The spectra show a diamond band peaking near $1322 \mathrm{~cm}^{-1}$ and about $30 \mathrm{~cm}^{-1}$ in bandwidth, not a very broad band peaking near $1200 \mathrm{~cm}^{-1}$ which has a bandwidth of several hundred inverse centimeters.

A more likely possibility is that the four-coordinated carbon has the structure of an amorphous form of diamond. Here there is an analogy to the silicon system. The Raman band of crystalline silicon peaks near $520 \mathrm{~cm}^{-1}$ with a narrow $\left(<5 \mathrm{~cm}^{-1}\right)$ linewidth, but its amorphous analog peaks at $465-480 \mathrm{~cm}^{-1}$ with a linewidth of 60 to $100 \mathrm{~cm}^{-1}$ [13]. A Raman spectrum of an amorphous silicon film on a crystalline silicon substrate is shown in Figure 6.

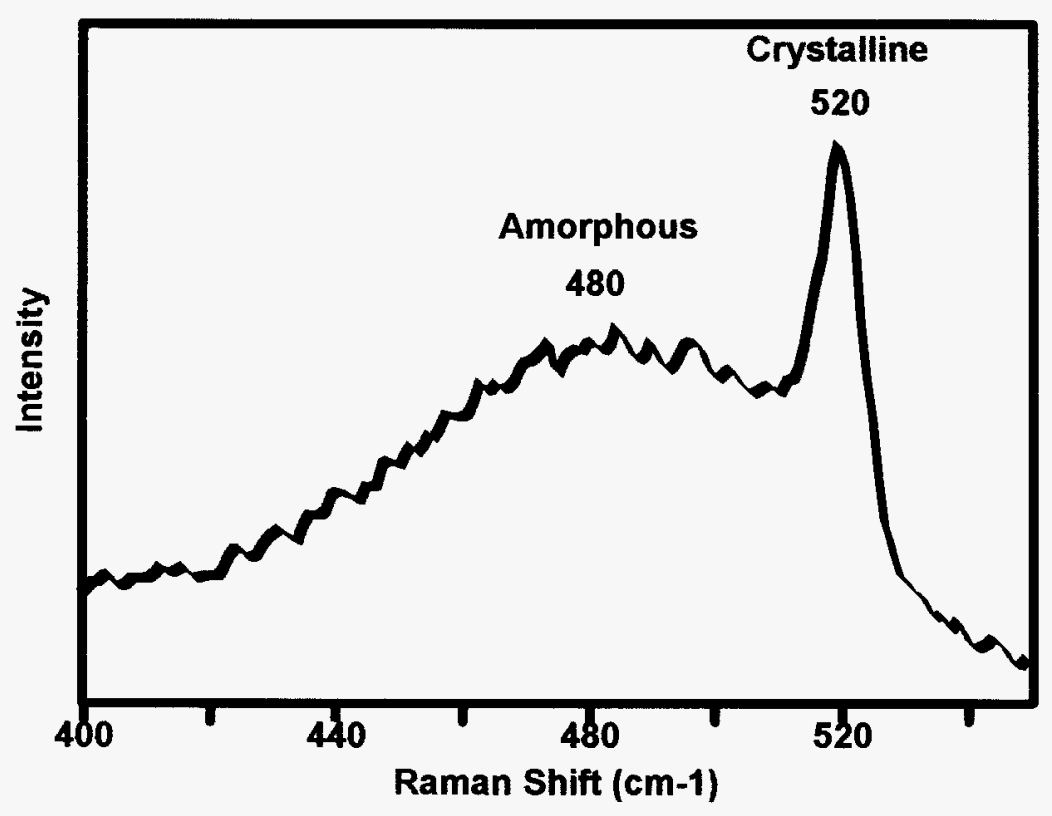

Figure 6. Raman spectrum of a film of amorphous silicon over a crystalline silicon substrate (514 $\mathrm{nm}$ excitation)

The Raman peak in silicon shifts downward from near $520 \mathrm{~cm}^{-1}$ to the vicinity of $480 \mathrm{~cm}^{-1}$ with a transition from a crystalline to an amorphous matrix. The decrease in 
frequency is due to an increase in bond lengths in the amorphous state. A proportional shift from the crystalline diamond peak $\left(1332 \mathrm{~cm}^{-1}\right)$ would result in an amorphous diamond band peaking near $1230 \mathrm{~cm}^{-1}$. Thus, the Raman band which is observed in ultraviolet-excited Raman spectra of amorphous carbon films and which increases in intensity with the amount of four-coordinated carbon is consistent with the Raman feature which would be expected from amorphous diamond.

\section{Three-Coordinated Carbon}

The complex changes in the Raman spectra of amorphous carbon films as a function of deposition conditions (see Figure 2) when the spectra are excited by visible wavelengths suggest that the structures incorporating three-coordinated carbon atoms may be large and complex. One possibility is that the three-coordinated carbon atoms are assembled into chains which have conjugated $\pi$-bonding. This possibility is supported by theoretical simulation $[4,6]$ and measurements of electronic transport [14], which suggest an average chain length of 13 atoms. Polyvinyl chloride (PVC) is a polymer which spontaneously generates conjugated polyene chains when it degrades by evolution of hydrogen chloride:

$$
\left(\mathrm{CH}_{2}-\mathrm{CHCl}\right)_{n} \rightarrow(\mathrm{CH}=\mathrm{CH})_{n}+\mathrm{HCl}
$$

Raman spectra of degraded PVC show resonance enhancement, with green excitation wavelengths, of bands from the conjugated chains when the chains reach about 17 polyene units in length $(n=17$, or 34 carbon atoms [15]). Two relatively narrow (about $30 \mathrm{~cm}^{-1}$ linewidth), approximately equal intensity, Raman bands appear resonantly enhanced in the spectra of degraded PVC. These bands peak about 1100 and $1500 \mathrm{~cm}^{-1}$. The Raman band pattern of PVC, with relatively narrow bands of equal intensity peaking near $1100 \mathrm{~cm}^{-1}$ and $1500 \mathrm{~cm}^{-1}$, cannot account for the Raman spectra of Figure 2, whose broad features peak from $1535 \mathrm{~cm}^{-1}$ to $1570 \mathrm{~cm}^{-1}$. We recognize that the Raman spectra in the PVC polyene units may be affected by the presence of hydrogen. However, even the Raman spectra of hydrogen-rich diamond-like (amorphous) carbon more closely resembles the Raman spectra of the low-depositionenergy films of Figure 2 than that of degraded PVC. It is probable that polyene-like chains exist in amorphous carbon films, but they don't appear to be the primary source of the amorphous carbon Raman bands.

Another possible set of structures incorporating three-coordinated carbon atoms include sheets made up of $\pi$-bonded rings composed of five, six or seven carbon atoms. Fullerenes and nanotubes [16] include these types of rings, with the sixmembered components forming flat sheets and the five- and seven-membered components providing curvature. These complex structures may include tens to hundreds of carbon atoms in sheets or ribbons, which may be too large to model with simulation protocols that use $100-300$ atoms per unit cell [4-6]. While no experimental confirmation of the presence of $\pi$-bonded rings exists, theoretical calculations [17] have predicted the Raman frequencies of five - to seven-membered, $\pi$-bonded carbon rings. Using a vibrational mode of $E_{2 g}$ symmetry peaking at $1569 \mathrm{~cm}^{-1}$ to represent sixmembered rings, plus modes of $A_{1}$. symmetry at $1444 \mathrm{~cm}^{-1}$ (five-membered rings) and $1303 \mathrm{~cm}^{-1}$ (seven-membered rings) and a small contribution from an $E_{2}$, mode at 1100 
$\mathrm{cm}^{-1}$ (five-membered rings), it is possible to reconstruct amorphous carbon Raman spectra over the range of deposition energies shown in Figure 2. Our fitting procedure used as adjustable parameters the intensities of the vibrational modes, with linewidths of 200 to $400 \mathrm{~cm}^{-1}$. Normalized integrated intensities of the calculated bands, fitted to spectra of amorphous carbon films deposited with energies from $5 \mathrm{~J} / \mathrm{cm}^{2}$ to $125 \mathrm{~J} / \mathrm{cm}^{2}$ (see Figure 2), are plotted in Figure 7.

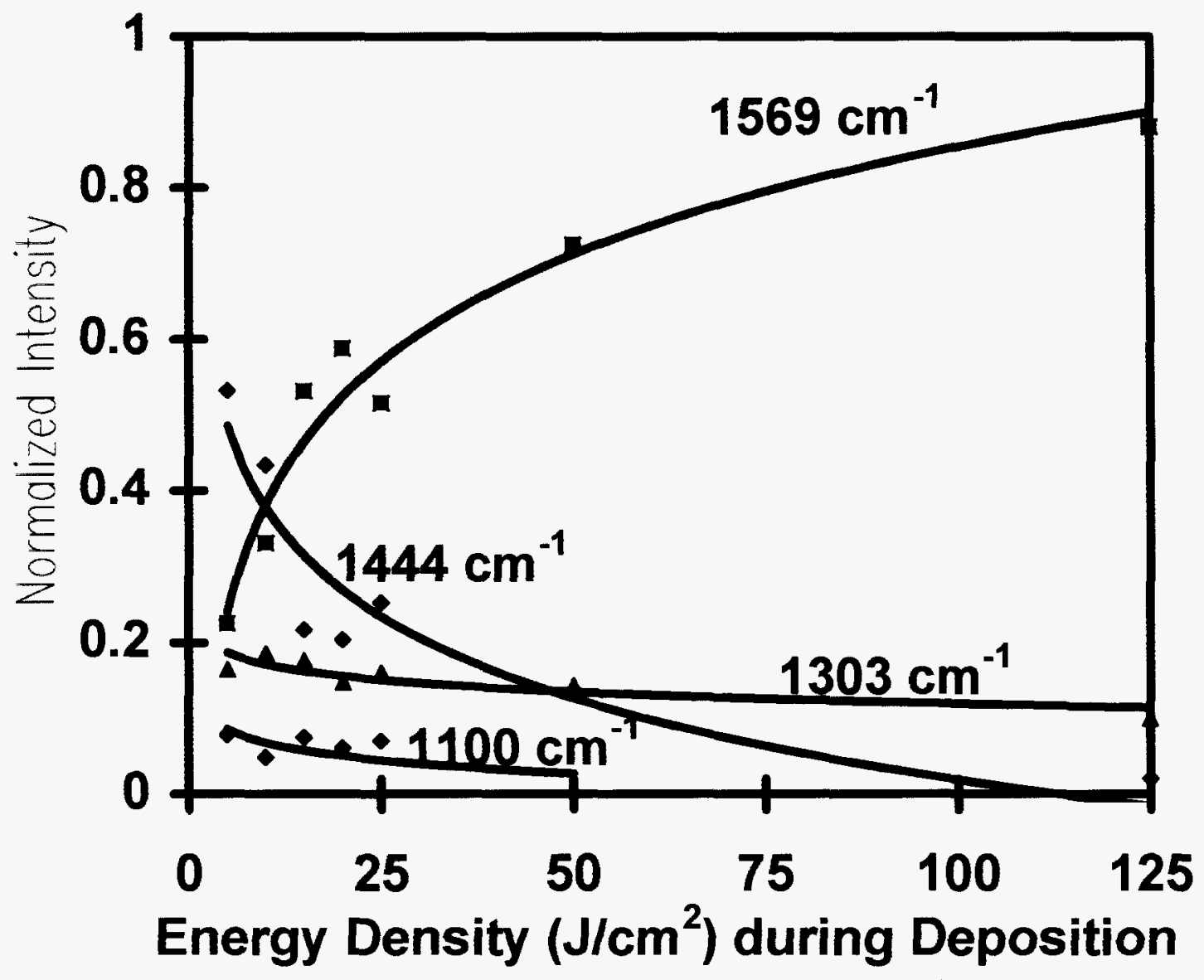

Figure 7. Normalized integrated intensities of the calculated vibrational modes fitted to Raman spectra of amorphous carbon films deposited at $5-125 \mathrm{~J} / \mathrm{cm}^{2}$. Squares - sixmembered rings $\left(1569 \mathrm{~cm}^{-1}\right)$; diamonds - five-membered rings $\left(1100 \mathrm{~cm}^{-1}\right.$ and $\left.1444 \mathrm{~cm}^{-1}\right)$; and triangles - seven-membered rings $\left(1303 \mathrm{~cm}^{-1}\right)$.

The results of the fitting procedure, as shown in Figure 7, indicate that, as the deposition energy increases, so does the proportion of six-membered rings at the expense of five- and seven-membered rings. Since the overall proportion of structures incorporating three-coordinated carbon atoms also decreases with increasing deposition energy, it appears that flat sheets of six-membered carbon rings are favored when the overall proportion of three-coordinated carbon atoms is low, and the threecoordinated carbon structures are likely to be smaller and more isolated. At low deposition energies and, consequently, a higher proportion of three-coordinated 
carbon, the larger and more densely packed three-coordinated carbon structures tend to incorporate more five- and seven-membered rings, which impart curvature to their surfaces. One can rationalize this behavior by considering the alternative to an increase in the proportion of five- and seven-membered rings with increasing three-coordinated carbon. Sheets of (flat) six-membered carbon rings, present in high density, can be expected to stack, forming graphite-like structures with a distinctive Raman band pattern (Figure 1), which has not been observed in these amorphous carbon films.

\section{CONCLUSION}

Because of resonance enhancement effects, the Raman band pattern obtained from amorphous carbon films depends on the laser excitation wavelength. Visible excitation wavelengths resonantly enhance structures incorporating $\pi$-bonded, threecoordinated carbon atoms, because their $\pi \rightarrow \pi^{*}$ transitions occur at visible wavelengths. Four-coordinated carbon structures have electronic transitions $\left(\sigma \rightarrow \sigma^{*}\right)$ in the far (vacuum) ultraviolet, and their Raman bands do not experience resonance enhancement with visible excitation. Bands from three-coordinated carbon structures dominate the Raman spectra with visible excitation. With ultraviolet excitation the enhancement of Raman bands from three-coordinated structures is not as pronounced, and a broad band peaking at 1100 to $1200 \mathrm{~cm}^{-1}$, which is due to four-coordinated carbon, becomes observable. The peak position and breadth of this band are consistent with an amorphous analog of diamond, and this Raman band is useful in following the relative proportion of four-coordinated versus three-coordinated carbon. The Raman bands of three-coordinated carbon structures, as obtained with visible excitation, have broad and complex patterns, which vary with deposition conditions. This variation in the Raman band patterns correlates to physical properties of the carbon film, such as density and internal stress, and it is apparently related to changes in the distribution of three-coordinated carbon structures. Calculated Raman spectra of small, $\pi$-bonded carbon rings reproduce the experimental data well, but conjugated chain structures may also be contributing to the observed Raman features.

Raman spectra of amorphous carbon are rich in information, much of which we do not yet know how to interpret. Definitive experiments which will completely characterize the structure of amorphous carbon have yet to be carried out.

\section{ACKNOWLEDGMENTS}

Sandia is a multiprogram laboratory operated by Sandia Corporation, a Lockheed Martin Company, for the United States Department of Energy under Contract DE-AC04-94AL85000. The authors thank Regina Simpson for obtaining the Raman spectra. 


\section{REFERENCES}

1. Xiong, Y. Y. Wang and R. P. H. Chang, Phys. Rev. B 48, p. 8016 (1993).

2. T. A. Friedmann, K. F. McCarty, J. C. Barbour, M. P. Siegal and D. C. Dibble, Appl. Phys. Lett. 68, 1643 (1996).

3. J. Robertson, Prog. Solid State Chem. 21, p. 199 (1991).

4. N. A. Marks, D. R. McKenzie, B. A. Pailthorpe, M. Bernasconi and M. Parrinello, Phys. Rev. Lett. 76, p. 768 (1996).

5. S. Nelson, E. B. Stechel, A. F. Wright, S. J. Plimpton, P. A. Schultz and M. P. Sears, Phys. Rev. B 52, p. 9354 (1995).

6. P. A. Schultz and E. B. Stechel, Phys. Rev. B (in press).

7. W. R. Gilkes, H. S. Sands, D. N. Batchelder, J. Robertson and W. I. Milne, Appl. Phys. Lett. 70, p.1980 (1997).

8. Y. Wang, D. C. Alsmeyer and R. L. McCreery, Chem. Mater. 2, p.557 (1990).

9. F. Tuinstra and J. L. Koenig, J. Chem. Phys. 53, p. 1126 (1970).

10. V. I. Merkulov, J. S. Lannin, C. H. Munro, S. A. Asher, V. S. Veerasamy and W. I. Milne, Phys. Rev. Lett. 78, 4869 (1997).

11. J. P. Sullivan, T. A. Friedmann and A. G. Baca, J. Electr. Mat. 26, p.1021 (1996).

12. M. Yoshikawa, Y. Mori, H. Obata, M. Maegawa, G. Katagiri, H. Ishida and A. Ishitani, Appl. Phys. Lett. 67, p.694 (1995).

13. F. H. Pollak, Test and Measurement World, p. 2 (May, 1985).

14. J. P. Sullivan and T. A. Friedmann, in Proc. First International Specialist Meeting on Amorphous Carbon, (World Scientific Publishing, Singapore, 1997), Cambridge, U. K., 1997.

15. W.F. Maddams, American Laboratory, p.59 (March, 1986).

16. See the cover and articles of the MRS bulletin, November, 1994.

17. T. E. Doyle and J. R. Dennison, Phys. Rev. B. 51, p. 196 (1995). 
M98002579

.

Report Number (14 )SAND $-998-0128 C$ CONF $-971201-$

publ. Date (11) 199801

sponsor code (18) DOE IDP, XF

JC Category (19) $C l e-700, D O E / E R$

DOE 